\title{
Distinct Stem Cells Subpopulations Isolated from Human Adipose Tissue Exhibit Different Chondrogenic and Osteogenic Differentiation Potential
}

\author{
Tommaso Rada • Rui L. Reis • Manuela E. Gomes
}

Published online: 16 April 2010

(C) Springer Science+Business Media, LLC 2010

\begin{abstract}
Recently adipose tissue has become a research topic also for the searching for an alternative stem cells source to use in cell based therapies such as tissue engineer. In fact Adipose Stem Cells (ASCs) exhibit an important differentiation potential for several cell lineages such as chondrogenic, osteogenic, myogenic, adipogenic and endothelial cells. ASCs populations isolated using standard methodologies (i.e., based on their adherence ability) are very heterogeneous but very few studies have analysed this aspect. Consequently, several questions are still pending, as for example, on what regard the existence/ or not of distinct ASCs subpopulations. The present study is originally aimed at isolating selected ASCs subpopulations, and to analyse their behaviour towards the heterogeneous population regarding the expression of stem cell markers and also regarding their osteogenic and chondrogenic differentiation potential. Human Adipose derived Stem Cells (hASCs)
\end{abstract}

T. Rada $\cdot$ R. L. Reis $(\bowtie) \cdot$ M. E. Gomes $(\bowtie)$

3B's Research Group-Biomaterials,

Biodegradables and Biomimetics,

Department of Polymer Engineering, University of Minho,

Headquarters of the European Institute of Excellence on Tissue

Engineering and Regenerative Medicine,

AvePark, 4806-909 Taipas,

Guimarães, Portugal

e-mail: rgreis@dep.uminho.pt

e-mail: megomes@dep.uminho.pt

URL: http://www.3bs.uminho.pt

T. Rada

e-mail: trada@dep.uminho.pt

URL: http://www.3bs.uminho.pt

T. Rada $\cdot$ R. L. Reis $\cdot$ M. E. Gomes

IBB - Institute for Biotechnology and Bioengineering,

PT Government Associated Laboratory,

Guimarães, Portugal subpopulations were isolated using immunomagnetic beads coated with several different antibodies (CD29, CD44, CD49d, CD73, CD90, CD 105, Stro-1 and p75) and were characterized by Real Time RT-PCR in order to assess the expression of mesenchymal stem cells markers (CD44, CD73, Stro-1, CD105 and CD90) as well as known markers of the chondrogenic (Sox 9, Collagen II) and osteogenic lineage (Osteopontin, Osteocalcin). The obtained results underline the complexity of the ASCs population demonstrating that it is composed of several subpopulations, which express different levels of ASCs markers and exhibit distinctive differentiation potentials. Furthermore, the results obtained clearly evidence of the advantages of using selected populations in cell-based therapies, such as bone and cartilage regenerative medicine approaches.

Keywords Adult stem cells · Mesenchymal stem cells . Cell separations - Cell isolation - Adipose tissue .

Cell differentiation

\section{Introduction}

Adipose-derived Stem Cells (ASCs) were firstly identified in 2001 by Zuk et al. [1] and since then ASCs have been the focus of many studies envisioning the better understanding of a number of cellular mechanisms (for instance involved in adipogenesis) $[2,3]$ and/or the application of these cells in a number of cell-based therapies, including Regenerative Medicine and Tissue Engineering [4-6]. In fact, the Adipose Tissue, mainly due to its wide availability and the relative simplicity of the harvesting procedures, presents a great potential as a source of adult stem cells for a number of applications as 
compared to other cell sources, such as for instance the bone marrow [7]. Furthermore, it is critical to develop therapies that involve a minimal time frame between the harvesting of the tissue and the application of the cells. This time consists in the summa of the expansion time to obtain a clinically relevant number of cells and eventually, for specific applications, the time needed to differentiate isolated cells. Obviously this time frame can be shortened by means of using adequate cell sources and/or specific harvesting/isolation methods and optimized culturing conditions. Until now, the most promising strategies in distinct stem cells therapies are based on the use of autologous cells and thus it is essential to optimize the number of cells that can be isolated from relatively small tissue samples of the patients. From the adipose tissue it is possible to obtain a high number of stem cells specially when compared with the bone marrow. In fact, the ratio between "Colonies-Forming-Unit Fibroblast" (CFU-F) and "Colonies-Forming-Unit expressing Alkaline Phosphatase" (CFU-AP) of the MSCs isolated from bone marrow is 1 in 100000 and 1 in 100 from ASCs isolated from an equal amount of Adipose Tissue [7, 8]. Nevertheless, the ASCs have obvious and profound similarities with the Bone Marrow MSCs (BM-MSCs) as both represent the stromal cell fraction of adipose tissue and bone marrow (both derived from the mesenchyme), respectively, and have similar morphology and surface proteins $[1,9,10]$. Therefore, most of the research work that can be found on ASCs is based on previous studies on BM-MSCs [11]. ASCs have also a great differentiation potential, demonstrated by a number of studies that have shown their neurogenic $[1,12,13]$, cardiomyocyte and myocyte [14-17], endothelial cells [1, 15], hepatocytes $[18,19]$, adipogenic $[1,20,21]$, and of course osteogenic and chondrogenic differentiation potential [22-26].

Most of the studies on ASCs that can be found in literature [27-32], describe the isolation of these cells using a method based on the enzymatic digestion of the adipose tissue with collagenase, followed by the seeding of the heterogeneous cell suspension, and subsequent removal of non-adherent cells. The principle of this isolation method is the property of the ASCs to adhere to the surface of the culture flasks/dishes and it obviously results in the presence of other cell types that may compromise the proliferation and/or differentiation potential of ASCs [30, 33-36]. The use of other methods may allow studying the possible presence of subpopulations, similarly to what has been found in the bone marrow [3740]. In fact several BM-MSC subpopulations have been studied in order to establish the phenotype, the gene expression and the proliferation rate as described in a study by Tormin et al. [39], which identified at least three different subpopulations; Gronthos et al. [41] were able to isolate and purify a $\mathrm{STRO}^{+} / \mathrm{VCAM}^{+} \mathrm{BM}-\mathrm{MSC}$ subpopulation that exhibited high proliferative properties. Studies on the differentiation potential of the several BM-MSC subpopulations have also been carried on by Satomura et al. [42], that showed that not all the stem cells subpopulations that were isolated from the bone marrow were able to differentiate into osteoblasts. Other studies suggested the presence of specific subpopulations in the bone marrow that have higher ability for the differentiation into the lipogenic lineage while other subpopulations present a superior ability for the osteogenic differentiation [37-39, 43-46]. These studies clearly demonstrate the presence of subpopulations in the BM-MSC and highlight the importance of developing isolation methods that allow obtaining selected cells populations and studing the characteristics that differ among them. However, a very limited number of such studies on ASCs is reported in the literature [47, 48]. Miranville et al [49] and Sengenes et al [48], with the objective of studying the neovascularisation and the angiogenesis in the adipose tissue, used a method that consisted in the selection of the ASCs using immunomagnetic beads coated with CD34 antibody followed by FACS analysis. This research group published several further studies $[47,48]$ where $\mathrm{ASCs} \mathrm{CD} 34^{+} / \mathrm{CD} 31^{+}$were isolated with the aim to study the vascularisation in the adipose tissue and the potential of these stem cells to differentiate into the endothelial lineage and they have demonstrated that at least one subpopulation of ASCs has this differentiation potential.

In this work, we hypothesised that there are of several subpopulations whitin the vascular fraction of the adipose tissue and consequently ASCs, obtained after enzymatic digestion, were selected using immunomagnetic beads coated with a range of antibodies, namely, CD29 ab, CD44 ab, CD49d ab, CD73 ab, CD90 ab, CD105 ab, p75 ab and STRO-1 ab. The selection of the majority of these antibodies was based on the ASCs gene expression profile, as it is know that ASCs express CD29, CD44, CD49d, CD90, CD73, and CD105 among others, such as CD49e and CD166 [7-9, 24, 34, 35]. STRO-1 was chosen because it has been used in previous studies to select subpopulation of BM-MSCs [40, 41, 50, 51] and p 75 was selected because it was the first marker to be commercialized for the isolation of BM-MSCs. The "stemness" [52] of the subpopulations was characterized through the analysis of the expression of MSC markers, as described by the Mesenchymal and Stem Cells Committee of the International Society for Cellular Therapy. The differentiation potential of the several subpopulations cultured using either osteogenic and chondrogenic inducing medium was also characterized through Real-Time RT-PCR and specific histological staining for osteogenic and chondrogenic differentiation, respectively. 


\section{Materials and Methods}

Harvesting and Isolation of Adipose Derived Stem Cells (ASCs)

Human subcutaneous adipose tissue samples were obtained from lipoaspiration procedures performed on women with ages between 35 and 45 years, under a protocol previously established with the Department of Plastic Surgery of Hospital da Prelada, in Porto, Portugal.

All the samples were processed within $24 \mathrm{~h}$ after the lipoaspiration; $300 \times \mathrm{g}$ circa of each sample were retrieved and processed for the isolation of hASCs. The lipoaspirate samples were firstly washed with a solution of PBS (Sigma Aldrich, Germany), 5 mg/500 mL Ciprobay 200 (Bayer, Germany) and 10\% Antibiotic-Antimycotic (Gibco, UK), and then digested with $0.2 \%$ collagenase type I A (Sigma Aldrich, Germany) in PBS for $60 \mathrm{~min}$ at $37^{\circ} \mathrm{C}$ under gentle stirring. The digested tissue was filtered using a $100 \mu \mathrm{m}$ filter mesh (Sigma Aldrich, Germany) and centrifugated at $800 \times \mathrm{g}$ for $7 \mathrm{~min}$ at $20^{\circ} \mathrm{C}$ and the supernatant removed. The obtained cells were resuspended in culture medium (basal medium) composed by D-MEM-LG (Sigma Aldrich, Germany), 10\% FBS (Gibco, UK) and 1\% AntibioticAntimycotic (Gibco, UK) and seeded in $75 \mathrm{~cm}^{2}$ culture flasks and incubated at $37^{\circ} \mathrm{C}$ with $5 \% \mathrm{CO}_{2}$ (cells corresponding to the Vascular Fraction, VF) or submitted to a method for selection of subpopulations as described bellow.

\section{Isolation of ASCs Using Immunomagnetic Beads}

To isolate subpopulations within the heterogeneous population of cells obtained after the enzymatic digestion as described above, it was optimized a method based on the use of immunomagnetic beads previously coated with different antibodies. The VF of each patient was divided in different fractions and for each fraction was performed a separation process with one of the antibodies selected. For this procedure it was selected the Dynal M-450 Epoxy beads (Dynabeads, Dynal Biotech, USA), which were prepared according to the instructions, provided by the manufacture (Dynal Biotech, USA). Briefly, the beads were coated, through $24 \mathrm{~h}$ incubation under gentle stirring in sodium phosphate buffer at room temperature, respectively with the anti human CD29 (Integrin, beta 1; Enzifarma, Portugal), CD44 (CD44 molecule (Indian blood group); Enzifarma, Portugal), CD49d (Integrin alpha 4; Serotec, USA), CD73 (5' nucleotidase, ecto; Enzifarma, Portugal), CD90 (Thy-1 cell surface antigen; eBioscience, USA), CD105 (Endoglin; eBioscience, USA), p75 (Nerve Growth Factor Receptor; ABCam, USA) or STRO-1 (Stromal Derived Factor 1; Santa Cruz Biotechnology, Inc., USA) antibody, using a concentration of $4 \mu \mathrm{g}$ of antibody for $1 \times 10^{7}$
Dynabeads. The cell pellet obtained upon the enzymatic digestion of the lipoaspirate samples was resuspended in a $0.1 \%$ BSA solution (Sigma Aldrich, Germany) in PBS (Sigma Aldrich, Germany) and then mixed with the Abcoated Dynabeads and incubated for $30 \mathrm{~min}$ at $4^{\circ} \mathrm{C}$, under gentle stirring, to promote the bonding between cells and the beads. Subsequently, the cells suspension and beads mixture was washed with PBS and $0.1 \%$ BSA and the cells bonded to the Ab-coated Dynabeads were separated from the remaining of the cell suspension using the Dynal MPC magnet (Dynal Biotech, USA). Finally, the cells bonded to the Ab-coated Dynabeads were seeded in $75 \mathrm{~cm}^{2}$ cell culture flasks and incubated at $37^{\circ} \mathrm{C}$ with $5 \% \mathrm{CO}_{2}$.

Characterization of the Cells Isolated Using

the Immunomagnetic Beads Coated

with Different Antibodies

All the cell populations isolated using the immunomagnetic beads coated with the different antibodies were cultured in $75 \mathrm{~cm}^{2}$ flask with basal medium, until confluence.

For evaluation of the expression of mesenchymal stem cells markers by the isolated subpopulations, as compared to the whole population obtained by the enzymatic procedure, Real-Time RT-PCR analysis was used to assess the expression of several markers known to be expressed by stem cells, such as CD44; CD73; STRO-1; CD105 and CD90. Furthermore, the isolated subpopulations were also cultured with specific chondrogenic and osteogenic medium in order to assess their differentiation potential into these lineages. Histological stainings and Real Time RT-PCR analysis were used to evaluate the ostogenic and chondrogenic differentiation potential, as it will be described in further sections.

\section{Expression of Mesenchymal Stem Cells Markers}

After reaching confluence ( $80 \%$ of total flask surface), all subpopulations were trypsinized and sub-cultured into $25 \mathrm{~cm}^{2}$ flasks for 10 days, using basal medium. The total RNA was extracted with TriZol Reagent (Invitrogen, USA) following the procedure provided by the supplier: briefly, $800 \mu \mathrm{l}$ of TriZol were added for each sample containing $1 \times 10^{6}$ cells. After an incubation of $5 \mathrm{~min}$, additional $160 \mu \mathrm{l}$ of chloroform (Sigma Aldrich, Germany) were added; the samples were then incubated for $15 \mathrm{~min}$ at $4^{\circ} \mathrm{C}$ and centrifuged at the same temperature and $13000 \times \mathrm{g}$ for $15 \mathrm{~min}$. After the centrifugation, the aqueous part was collected and an equal part of isopropanol (Sigma Aldrich, Germany) was added. After an incubation of $2 \mathrm{~h}$ at $-20^{\circ} \mathrm{C}$ the samples were washed in ethanol, centrifuged at $4^{\circ} \mathrm{C}$ and $9000 \times \mathrm{g}$ for $5 \mathrm{~min}$ and resuspended in $12 \mu \mathrm{l}$ of water RNase/DNase free (Gibco, UK). The samples were quantified using the NanoDrop ND-1000 Spectrophotometer 
(NanoDrop Technologies, USA). For the cDNA synthesis the samples with a 260/280 ratio between 1.7 and 2.0 were used. The cDNA synthesis was performed using the iScript cDNA synthesis Kit (BioRad, USA), and the MiniOpticom Real-Time PCR Detection System (BioRad, USA), using an initial amount of total RNA of $2 \mu \mathrm{g}$ and a total volume of $20 \mu \mathrm{l}$. RNAse free water (Gibco, UK) was used as a negative control.

After the synthesis of the cDNA, the cells cultured with D-MEM were investigated regarding the expression of the genes CD73, STRO-1, CD105, CD90, and CD44. For each sample, GAPDH was used as housekeeping gene. The primers have been designed using the Primer 3 online software ( $\mathrm{v}$ 0.4.0) and synthesized by MWG Biotech Germany (Table 1).

The Real Time PCR was carried out using iQ Syber Green SuperMix equipment (BioRad, USA); reactions were performed following the procedure suggested by the manufacturer. To analyze the obtained data it was used the Delta Delta $\mathrm{Ct}$ method. The Delta Delta $\mathrm{Ct}$ was calculated using as reference sample the results obtained from the stromal vascular fraction. The results calculated as $2-\Delta \Delta C t$ and were elaborated using the Origin Pro 7.0 (OriginLab Corporation, USA) and the correlation between the dataset from the same gene analyzed was investigated with the ANOVA single factor method.

\section{Assessment of the Osteoblastic Differentiation}

For assessment of the osteoblastic differentiation potential of the cells isolated using the different procedures described, the cells were seeded in $25 \mathrm{~cm}^{2}$ flasks and cultured using basal medium (as control), and osteogenic medium composed of alpha modified eagle medium ( $\alpha$-MEM, Sigma Aldrich, Germany), supplemented with $50 \mu \mathrm{g} / \mathrm{ml}$ ascorbic acid (Sigma Aldrich, Germany), 10-8 M dexamethasone (Sigma Aldrich, Germany), $10 \mathrm{mM} \beta$-glycerol phosphate (Sigma Aldrich, Germany), 10\% FBS (Gibco, UK) and 1\% antibiotic/antimycotic (Gibco, UK).
After 3 weeks of culture with osteogenic medium, samples were retrieved for alizarin red-S staining and Real Time RT-PCR analysis for osteopontin and osteocalcin.

\section{Alizarin Red Staining}

The cells were fixed with formalin (Sigma Aldrich, Germany) and washed first with PBS and then with distilled water. Then the cells were stained with a $2 \%$ Alizarin Red solution (Merck, Germany) in distilled water for $5 \mathrm{~min}$, and finally washed with distilled water. Cultures were observed under an inverted microscope (Axivert 40 CFL, Zeiss, Germany).

\section{Osteopontin and Osteocalcin Expression}

Osteopontin and osteocalcin expression was assessed through Real Time RT-PCR analysis, which was performed as described for the analyses of mesenchymal stem cell markers expression. In this case the Delta Delta $\mathrm{Ct}$ was calculated using the osteopontin and osteocalcin expression of the cells cultured with basal medium as control. The primers used were reported in Table 1.

\section{Assessment of the Chondrogenic Differentiation Potential}

For the assessment of the chondrogenic differentiation potential, the cells were cultured in the form of pellets (obtained by centrifugation at $800 \mathrm{~g}$ of $5 \times 10^{6}$ cells). For each subpopulation, 6 pellets have been cultured with chondrogenic medium composed of D-MEM (Sigma Aldrich, Germany) supplemented with $1 \mathrm{mM}$ dexamethasone (Sigma Aldrich, Germany), 0.1 M sodium pyruvate (Sigma Aldrich, Germany), $17 \mathrm{mM}$ ascorbic acid (Sigma Aldrich, Germany), 35 mM proline (Fluka, Germany), ITS (Sigma Aldrich, Germany), 1\% antibiotic/antimycotic (Gibco, UK), 10 ng/ml TGF- $\beta 1$ (Pepro-Tech, USA) and $10 \%$ FBS (Gibco, UK) for 3 weeks in $15 \mathrm{ml}$ Falcons tube. At the end of the third week of culture, the pellets were

Table 1 Summary of the primers used in this study to perform RT Real Time PCR

\begin{tabular}{llll}
\hline Primer name & Sequence FW & Sequence RW & NCBI Reference sequence \\
\hline GAPDH & GCGACGGCTATGATTACGAT & ACTTGGAGGCAGTCCAGAGA & NM_002046.3 \\
CD105 & TCCTCCCAAGGACACTTGTA & CGCCTCATTGCTGATCATAC & NM_001114753 \\
CD44 & GTTGCCAAACCACTGTTCCT & CATTCAAATCCGGAATGCT & NM_000610 \\
STRO-1 & GAAGCTAAAGTGGATTCAGGAGTA & TAAGCAGGGGACCATTACA & DQ345517 \\
CD73 & CGCAACAATGGCACAATTAC & CTCGACACTTGGTGCAAGA & NM_002526 \\
Osteocalcin & GGCAGCGAGGTAGTGAAGAG & CTGGAGAGGAGCAGAACTGG & NM_099173 \\
Osteopontin & CCCACAGACCCTTCCAAGTA & GGGGACAACTGGAGTGAAAA & NM_001040058 \\
Collagen II & GACAATCTGGCTCCCAAC & ACAGTCTTGCCCCACTTAC & NM_001844 \\
Sox 9 & TACGACTACACCGACCACCA & TTAGGATCATCTCGGCCATC & NM_000346 \\
\hline
\end{tabular}


collected and were used for histological analysis (toluidineblue and safranin-O staining) and for Real Time RT-PCR for Collagen II and SOX 9.

\section{Toluidine-Blue and Safranin-O Staining}

The pellets were fixed in formalin and embedded in paraffin. The pellets were cut into sections of $8 \mu \mathrm{m}$, fixed on a slide and deparaffinated. Sections were stained by adding one drop of $1 \%$ toluidine blue onto each section for 2-3 s; and then safranin-O staining was also performed adding one drop of $0.1 \%$ safranin-O solution onto each section for $5 \mathrm{~min}$. In both cases, the stained sections were subsequently rinsed with distilled water and dehydrated using 100\% ethylene alcohol and allowed to dry overnight. The stained samples were analysed under a optical Imager. Z1 fluorescence microscope (Axivert 40 CFL; Zeiss, Germany) and photographed using an Axio Cam MRm camera (Zeiss, Germany).

\section{Collagen II and Sox 9 Expression}

Collagen II, Sox 9 and Aggrecan expression was assessed on cell pellets cultured for 3 weeks in chondrogenic medium, performing the Real Time RT-PCR as described in "Expression of Mesenchymal Stem Cells Markers" section. The primers used were reported in Table 1.

The Delta Delta Ct was calculated using the collagen II, sox 9 and aggrecan expression of the cells cultured with Basal Medium as control.

\section{Statistical Analysis}

Data are presented as arithmetic mean $\pm \mathrm{SD}$. An ANOVA Single Factor Test was used to compare the mean values of the results obtained from Real-Time RT-PCR analysis. The reference sample considered was the Vascular Fraction (VF). Differences were considered to be significant for $p<$ 0.05 . All statistical tests were performed using Origin Pro 7.0 (OriginLab Corporation, USA).

\section{Results}

Stem Cells Characterization: Real Time RT-PCR

The results obtained from the Real Time RT-PCR analysis regarding the expression of STRO-1, CD44, CD73, CD90 and $\mathrm{CD} 105$ in each of the isolated subpopulations are summarized in Table 2. The results showed in the Fig. 1 depict a complex scenario where not all the isolated subpopulations express the same gene profile and/or where not all the gene expression levels are higher than the
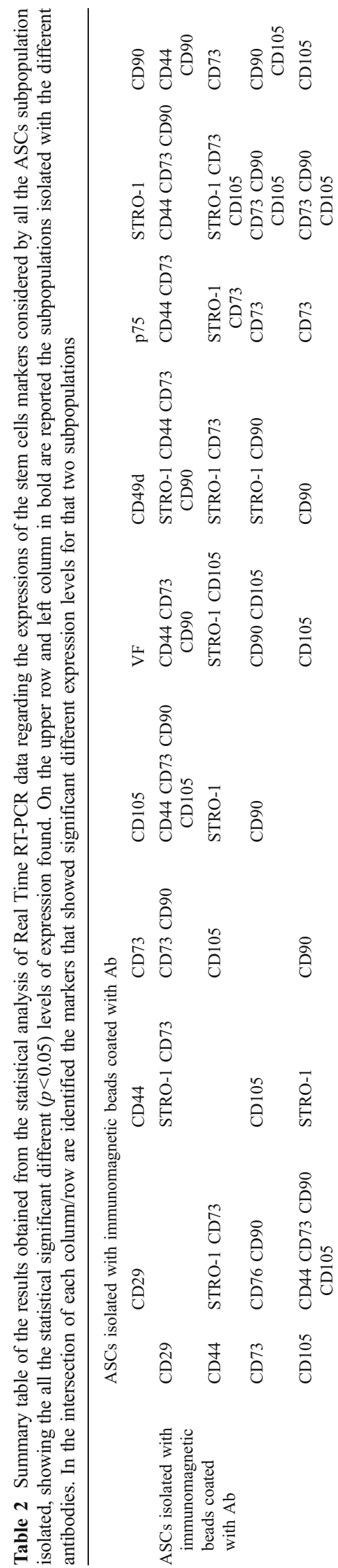

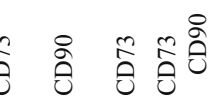

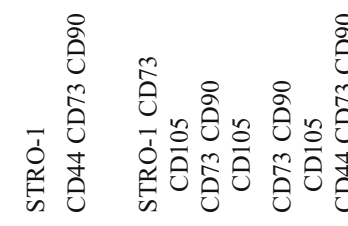

$$
\text { 西 }
$$

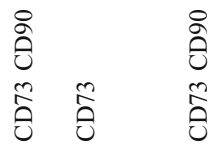

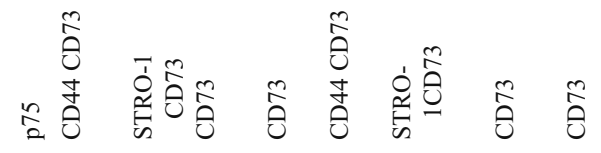

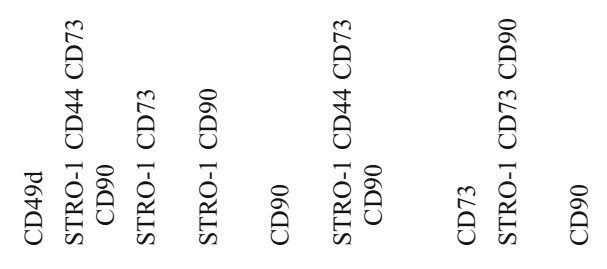

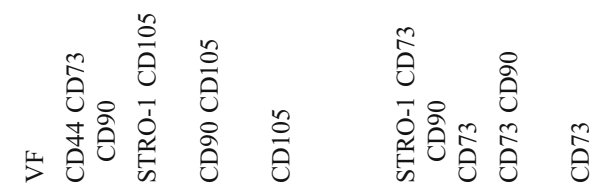

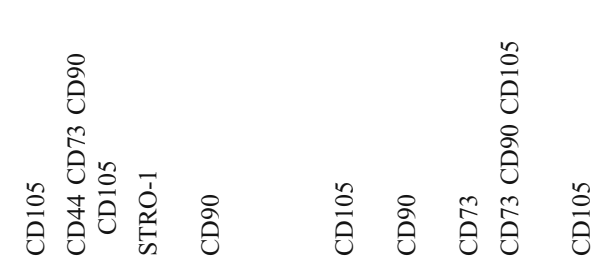

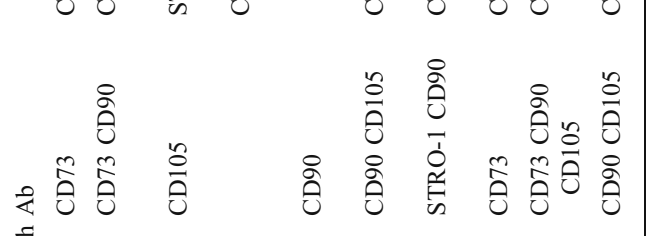
营

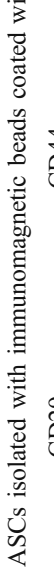

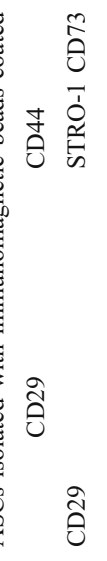

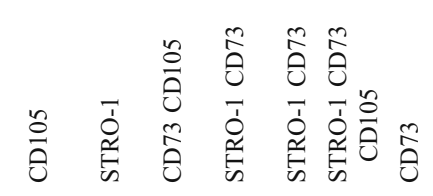

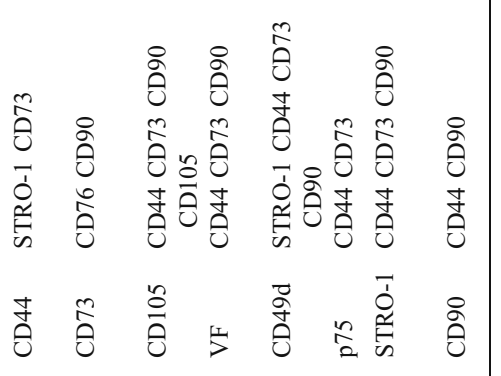

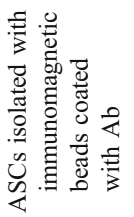

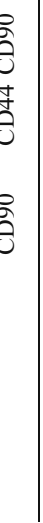



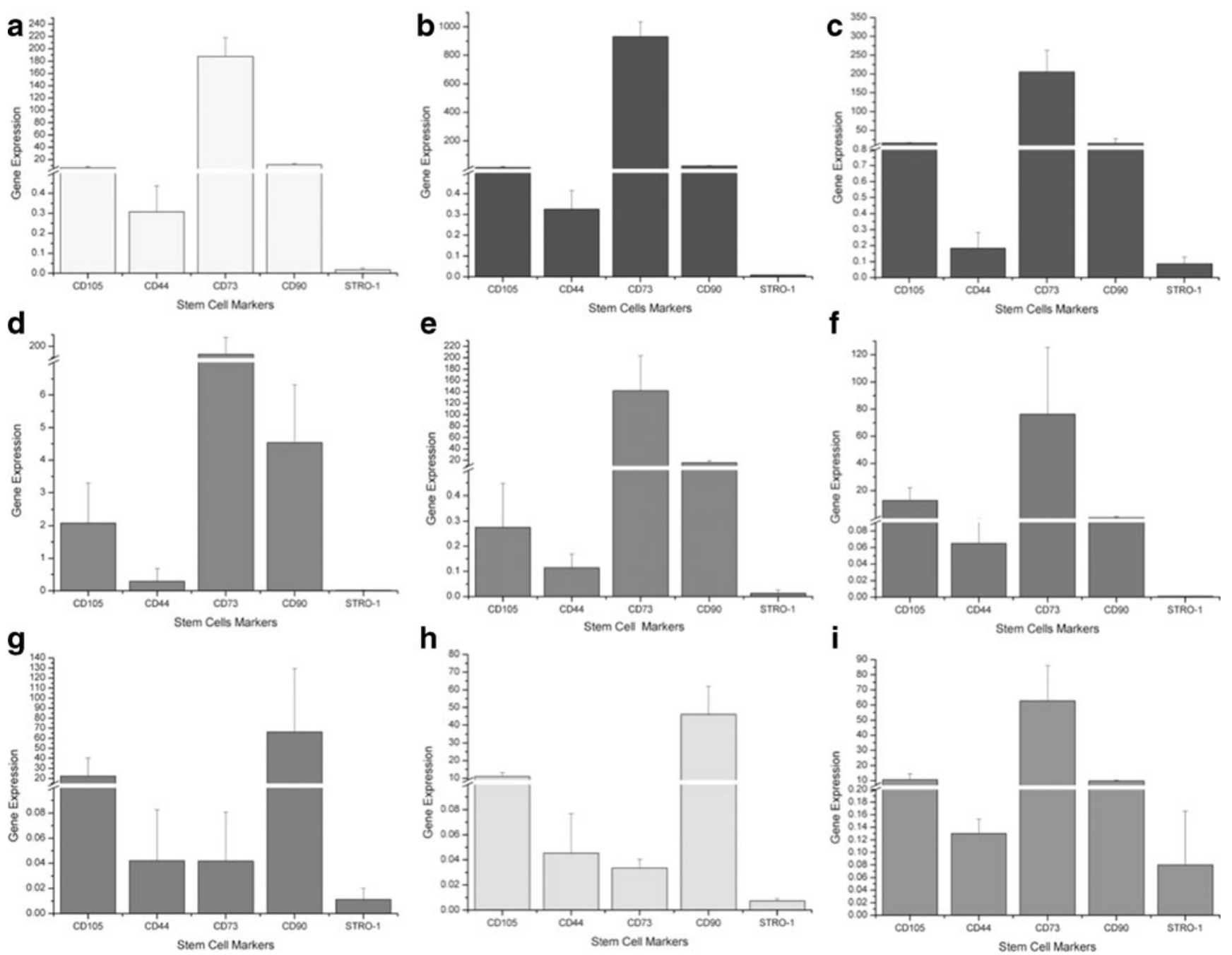

Fig. 1 Graphical representation of the Real Time RT-PCR analysis results of the various genes analysed by the ASCs isolated with the enzymatic digestion only (a) and by the subpopulations isolated using the immunomagnetic beads coated with the different antibodies, namely: b ASCs isolated with anti CD29 antibody; c ASCs isolated

with anti CD44 antibody; d ASCs isolated with anti CD73 antibody; e ASCs isolated with anti CD105 antibody; f ASCs isolated with anti CD49d antibody; g ASCs isolated with anti p75 antibody; h ASCs isolated with anti STRO-1 antibody; i ASCs isolated with anti CD90 antibody; (ANOVA single factor; $p<0.05$ )

vascular fraction (VF) represented by the baseline. Nevertheless all the subpopulations isolated showed clearly a lower expression of STRO-1, CD105 and CD44 compared with the VF, while in what concerns to the gene CD73 only the subpopulation isolated with the immunomagnetic beads coated with p75 and STRO-1 ab, presented a lower expression than that registered for the VF.

\section{Osteogenic Differentiation Potential}

\section{Alizarin Red Staining}

After 3 weeks of culture with osteogenic medium, all the cells subpopulations isolated with the different antibodies were positively stained for Alizarin Red, as it showed in Fig. 2, demonstrating the occurrence of the mineralization process. However, cell populations obtained from the enzymatic digestion (VF) (data not shown) and the subpopulation isolated with the immunomagnetic beads coated with anti STRO-1 Ab showed a more intensive staining, indicating the accuracy of a more widespread mineralization process.

\section{Real Time RT-PCR for Osteocalcin and Osteopontin}

After the osteogenic differentiation, Real Time RT-PCR analysis was performed in order to study the expression of osteopontin and osteocalcin, an early and a late marker of the osteogenic differentiation, respectively 

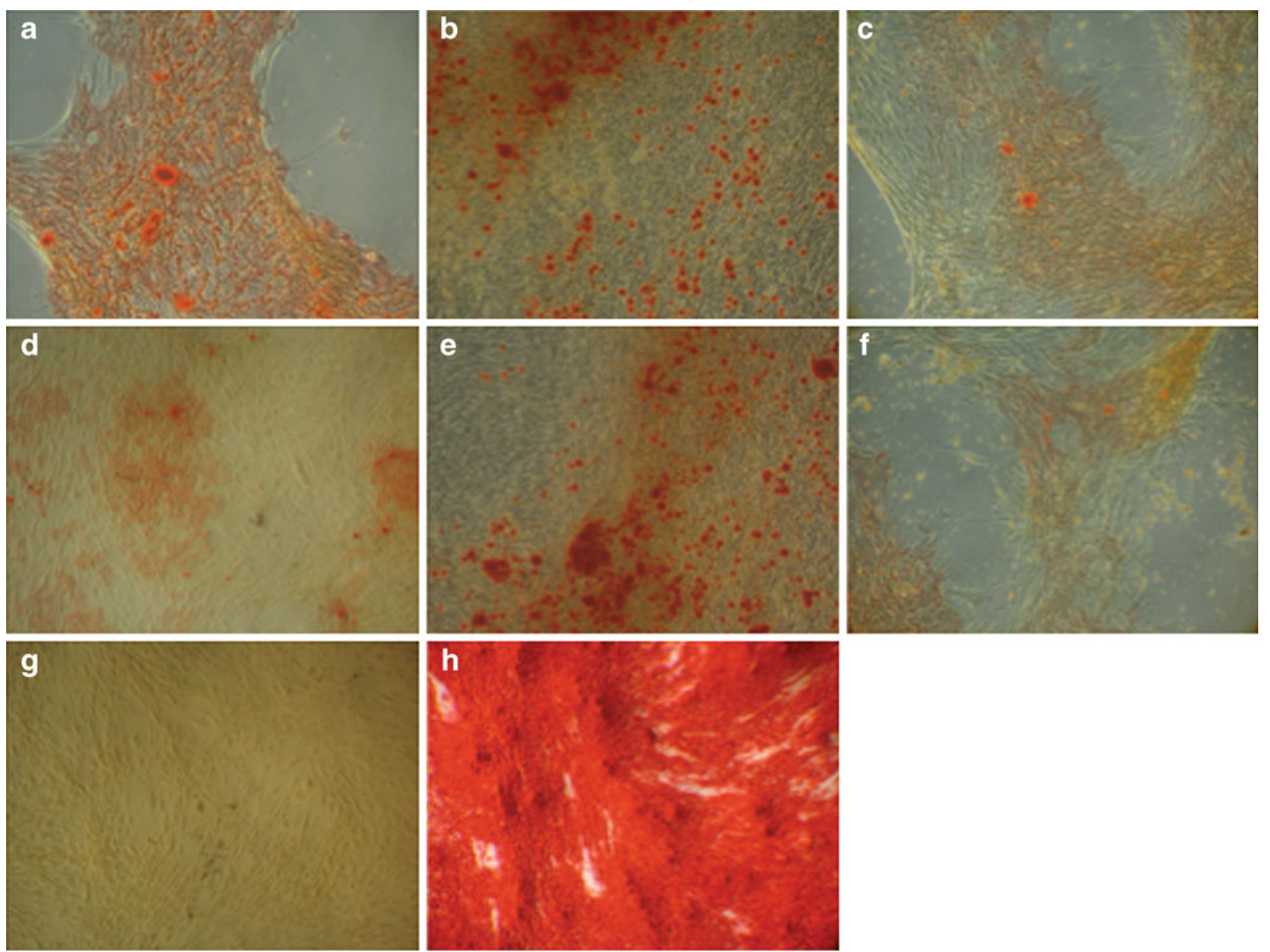

Fig. 2 Optical microscope pictures showing the cells obtained using the different isolation procedure stained, cultured with osteogenic medium and stained for alizarin red: a human ASCs isolated with immunomagnetic beads coated with anti CD29 ab $(100 \times)$; b human ASCs isolated with immunomagnetic beads coated with anti CD44 ab (100×); c human

ASCs isolated with immunomagnetic beads coated with anti CD49d ab (100×); d human ASCs isolated with anti p75 ab (100×); e human ASCs isolated with anti CD105 ab (100×); $\mathbf{f}$ human ASCs isolated with anti CD73 ab (100×); g human ASCs isolated with anti CD90 ab $(100 \times)$; $\mathbf{h}$ human ASCs isolated with anti STRO-1 ab $(100 \times)$

$[53,54]$. The results obtained are reported in Fig. 3 while the statistical analysis are reported in Table 3 and, in general, show a down regulation of osteopontin contrasted by an up regulation of osteocalcin. These results are in agreement with the images obtained from the Alizarin red stained sections, and confirm the occurrence of the osteogenic differentiation process and subsequent mineralization.

However only few of these isolated subpopulations have an higher expression rate of osteopontin in respect to the Vascular Fraction; namely the ASCs subpopulation isolated with anti CD49d ab; with anti CD90 antibody and with anti p75 antibody.

Regarding the expression of osteocalcin, all the subpopulation isolated expressed this gene. Furthermore in all the cases the expression of osteocalcin is at least ten times higher than osteopontin, demonstrating that the

differentiation process has already reached an advanced stage. This is particularly evident in the cells isolated with the antibody anti STRO-1 coated beads, that showed an intensive staining in the alizarin red assay and simultaneously a quite low expression of osteocalcin, suggesting that the differentiation process of this cells subpopulation is so advanced that the expression of this gene started to drop down.

\section{Chondrogenic Differentiation Potential}

\section{Toluidin Blue and Safranin-O Staining Assay}

After 3 weeks of pellet culture with chondrogenic medium all the isolated subpopulations were positively stained with toluidin blue and safranin-O showing the production of extracellular matrix (see Fig. 4). 

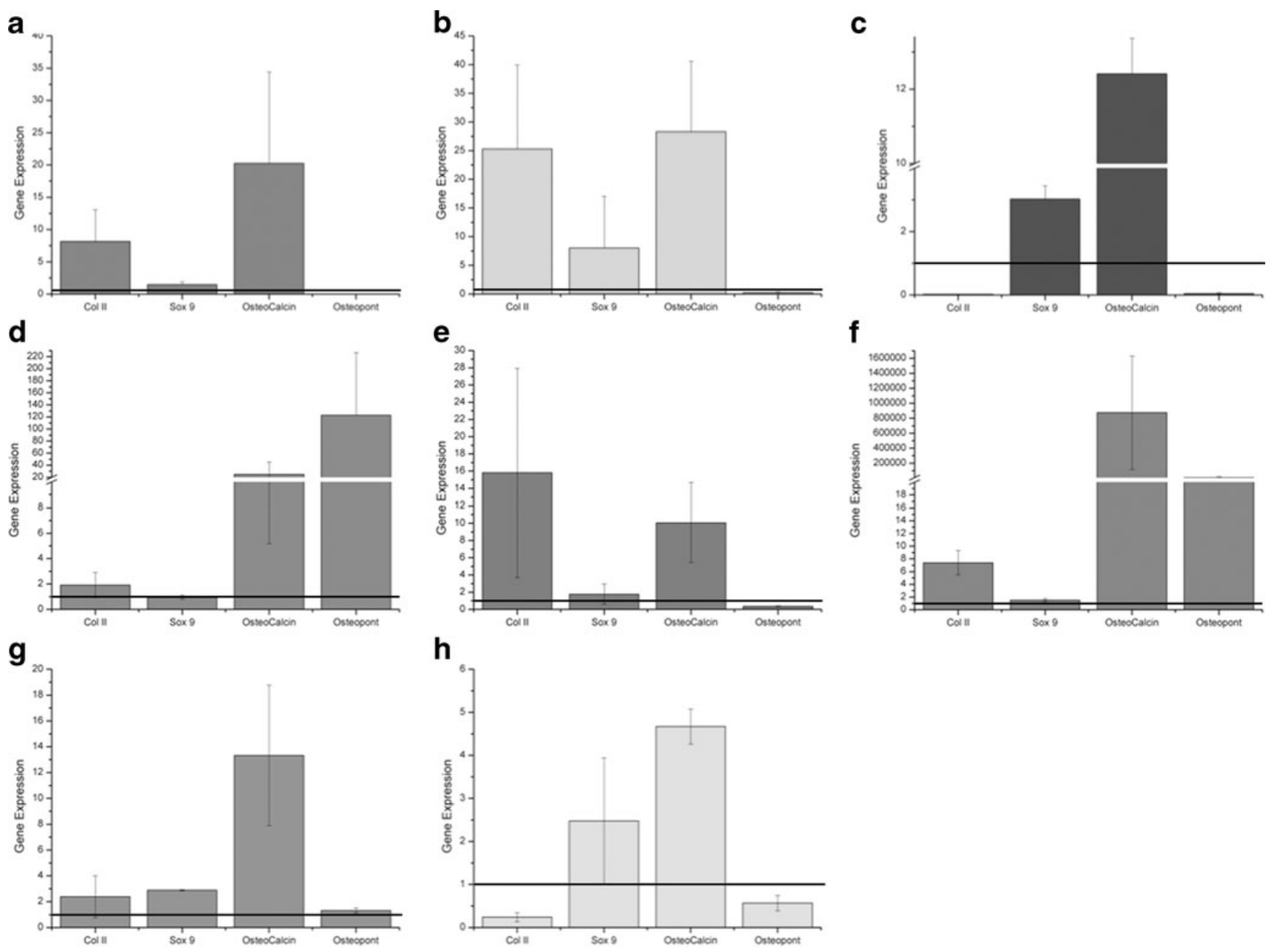

Fig. 3 Graphical representation of the results obtained from Real Time RT-PCR analysis regarding the expression of different chondrogenic and osteogenic marker genes according to the ASCs isolation procedure used, namely a ASCs isolated with immunomagnetic beads coated with anti CD105 antibody; b ASCs isolated with immunomagnetic beads coated with anti CD29 antibody; c ASCs isolated with immunomagnetic beads coated with anti CD44 antibody; d ASCs

\section{Real Time RT-PCR for Collagen II and Sox 9}

After 3 weeks of culture with chondrogenic medium, Real Time RT-PCR was performed in order to evaluate the expression of Sox 9 and collagen II, in each one of the isolated subpopulations. Sox 9 is a regulatory early marker of the chondrogenic differentiation process and collagen II is a well known marker of the extracellular matrix and therefore usually expressed at the end of the chondrogenic process $[55,56]$. The results are reported in the Fig. 3 while the statistical analysis are reported in Table 3 . All the cells isolated with the immunomagnetic beads coated with the antibodies used, except the cells CD49d+, express Collagen II. Sox 9 was also found to be expressed by all the subpopulations isolated. isolated with immunomagnetic beads coated with anti CD49d antibody; e ASCs isolated with immunomagnetic beads coated with anti CD73 antibody; f ASCs isolated with immunomagnetic beads coated with anti CD90 antibody; g ASCs isolated with immunomagnetic beads coated with anti p75 antibody; h ASCs isolated with immunomagnetic beads coated with anti STRO-1 antibody. Results analysed using ANOVA single factor; $p<0.05$

Nevertheless, the cell subpopulations studied showed different expression levels of the chondrogenic genes considered. In fact, the cell subpopulations that showed the highest expression of the Sox 9 gene, were those isolated with the immunomagnetic beads coated with anti $\mathrm{CD} 44 \mathrm{Ab}, \mathrm{p} 75 \mathrm{Ab}$ and $\mathrm{CD} 105 \mathrm{Ab}$, while regarding the expression of collagen II, the highest levels of expression were detected in the cell subpopulations isolated with anti CD29 Ab, CD73 Ab and CD105 Ab.

\section{Discussion}

To our best knowledge the present study reports, for the first time, the identification of a number of subpopula- 
Table 3 Summary table of the results obtained from the statistical analysis of Real Time RT-PCR data regarding the expression of the differentiation marker considered by all the ASCs subpopulations isolated, showing the all statistical significant different $(p<0.05)$ levels of expression found. On the upper row and left column in bold are reported the subpopulations isolated with the different antibodies. In the intersection of each column/row are identified the markers that showed significant different expression levels for that two subpopulations

\begin{tabular}{|c|c|c|c|c|c|c|c|c|c|}
\hline \multirow[t]{7}{*}{$\begin{array}{l}\text { ASCs isolated with } \\
\text { immunomagnetic beads } \\
\text { coated with } \mathrm{Ab}\end{array}$} & $\begin{array}{l}\text { CD105 } \\
\text { CD29 }\end{array}$ & CD105 & CD29 & $\begin{array}{l}\text { CD44 } \\
\text { Col II Sox } 9 \\
\text { Col II }\end{array}$ & CD49d & $\begin{array}{l}\text { CD73 } \\
\text { OP }\end{array}$ & CD90 & $\begin{array}{l}\text { P75 } \\
\text { OP Sox } 9 \\
\text { OP }\end{array}$ & $\begin{array}{l}\text { Stro-1 } \\
\text { OP Col II } \\
\text { OC }\end{array}$ \\
\hline & CD44 & Col II Sox 9 & Col II & & Col II Sox 9 & $\mathrm{OP}$ & Col II Sox 9 & OP OC & Col II OP OC \\
\hline & CD49d & & & Col II Sox 9 & & & Col II Sox 9 & Sox 9 & \\
\hline & CD73 & $\mathrm{OP}$ & & $\mathrm{OP}$ & & & & OP OC & \\
\hline & CD90 & & & Col II Sox 9 & Col II Sox 9 & & & Col II & \\
\hline & $\mathrm{p} 75$ & OP Sox 9 & $\mathrm{OP}$ & OP OC & Sox 9 & OP OC & Col II & & OP \\
\hline & Stro-1 & OP Col II & $\mathrm{OC}$ & Col II op OC & & & & OP & \\
\hline
\end{tabular}
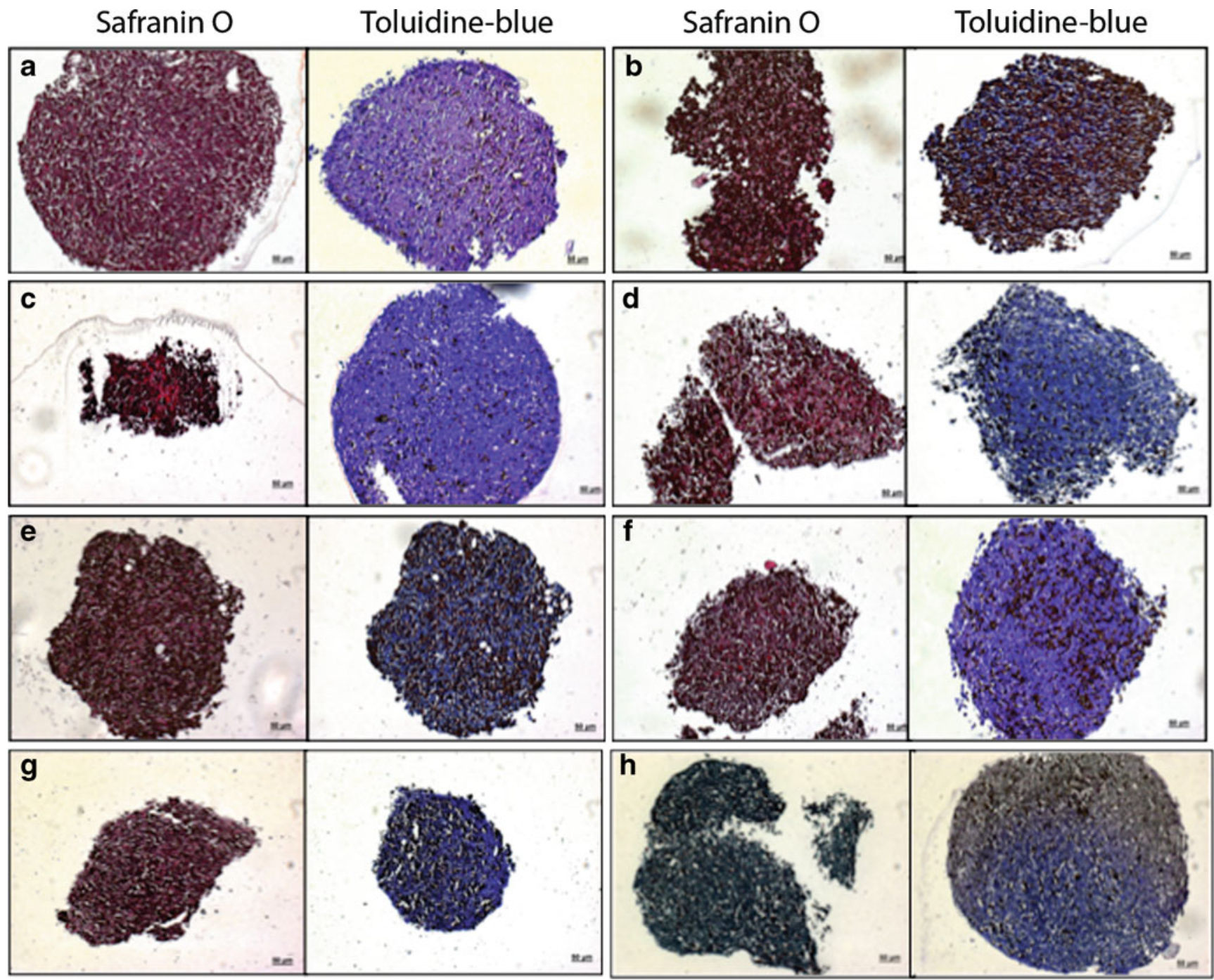

Fig. 4 Optical microscope pictures showing the cells obtained using the different isolation procedure, cultured in chondrogenic medium and stained for Safranin-O and Toluidin Blue a hASCs isolated with immunomagnetic beads coated with anti CD29 ab (100×); b hASCs isolated with immunomagnetic beads coated with anti CD44 ab

$(100 \times)$; $\mathbf{c}$ hASCs isolated with immunomagnetic beads coated with anti CD49d ab (100×); d hASCs isolated with anti CD73 ab (100×); e hASCs isolated with anti CD90 ab (100×); f hASCs isolated with anti CD105 ab $(100 \times)$; g hASCs isolated with anti STRO-1 ab $(100 \times)$; h hASCs isolated with anti p75 ab $(100 \times)$ 
a

Osteopontin Gene Expression

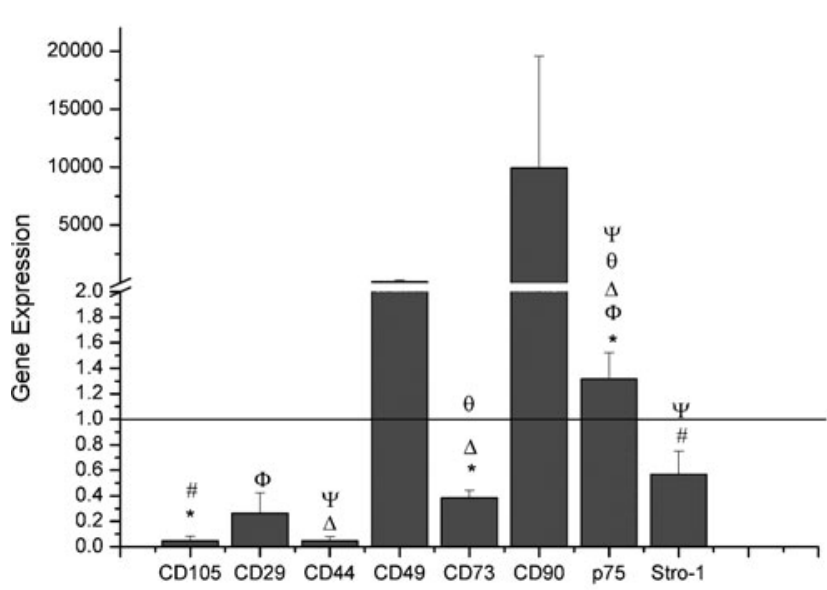

C

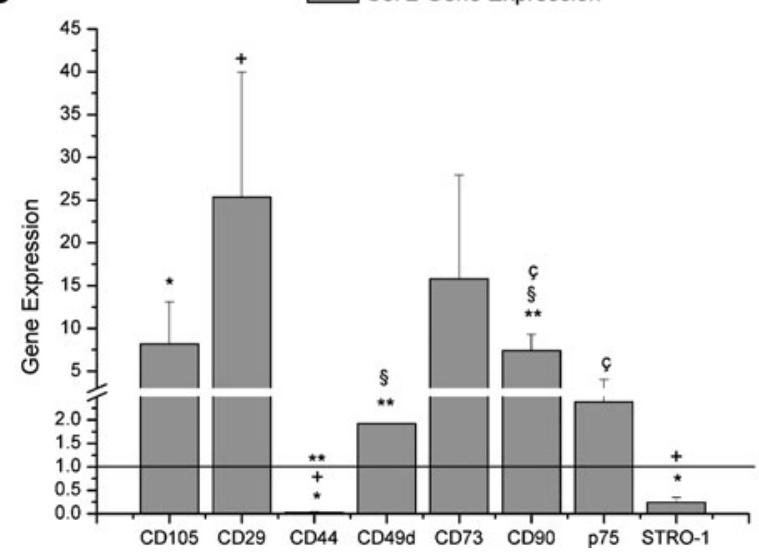

Fig. 5 Graphical representation of the results obtained from Real Time RT-PCR analysis regarding the expression of different chondrogenic and osteogenic marker genes of the different hASCs subpopu-

tions of adult adipose tissue derived stem cells. ASCs subpopulations were isolated using immunomagnetic beads coated with different antibodies and then it was determined the expression level of genes that have been considered in many studies as markers for the adipose tissue-derived stem cells. Moreover the ASCs isolated subpopulations were tested regarding their osteogenic and chondrogenic differentiation potential, envisioning their target application in osteo/cartilage tissue engineering applications.

For a more comprehensive discussion of the results obtained, it is necessary to analyse separately the expression of the stem cell markers genes and the data obtained from the differentiation potential experiments. The expression of the considered typical markers of ASCs shows that in the ASCs population (resulting from the enzymatic digestion only) co-exist several subpopulations that present some common markers expression, such as in the case of the cells isolated with immunomagnetic beads coated with anti CD29 and CD73

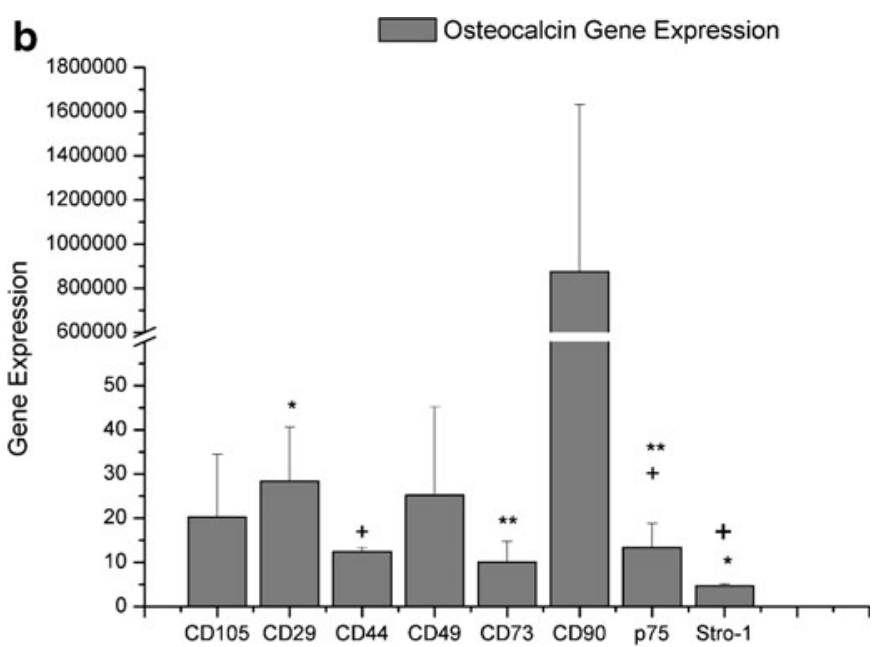

d

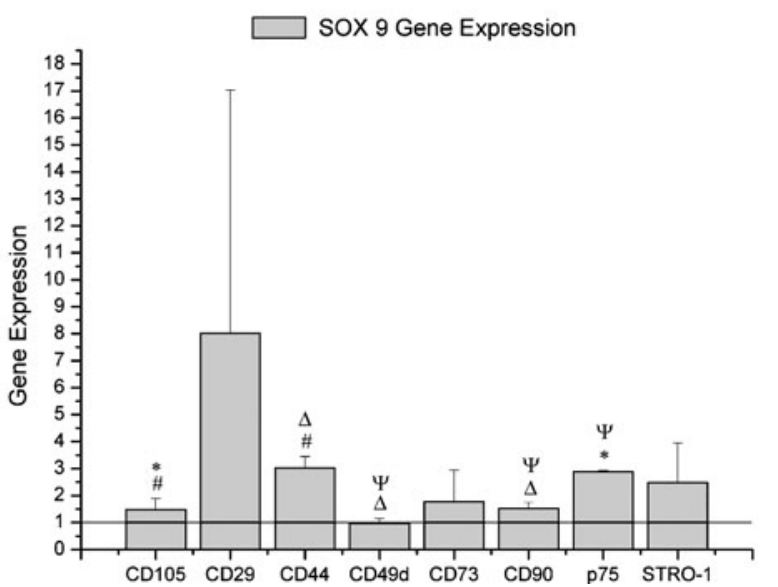

lations isolated a Osteopontin gene expression; b Osteocalcin Gene Expression; c Collagen II Gene Expression; d Sox 9 Gene Expression. Results analysed using ANOVA single factor; $p<0.05$

antibodies, that are not statistically significant different in the expression of CD105 and CD44 markers but show statistically different expression level of the CD73 and CD90 markers, for example.

The example described above can be applied to all the cell subpopulations isolated and the differences/analogies between the several cell subpopulations are summarized in the Table 2, where all the statistically differences found between the distinct subpopulations are summarized.

Nevertheless, in all the ASCs subpopulations, except the one isolated with the immunomagnetic beads coated with anti STRO-1 ab and anti p75 ab, it is possible to observe a common signature, where even if the level of expression differs, the ratio between the analyzed markers is conserved. In fact, the CD73 is the highest marker to be expressed followed by CD105; CD90; CD44 and STRO-1. As mentioned, the subpopulations isolated with anti STRO-1 $a b$ and p75 ab differs from all the other showing the highest expression with CD90 followed by CD105; CD44; CD73 and STRO-1. 
The assays performed to assess the chondrogenic and osteogenic differentiation potential show that all the subpopulations isolated can differentiate into these two lineages but the subpopulations have different differentiation potential (Fig. 5).

In fact, even if the alizarin red staining is positive for all the subpopulations isolated, as well as the expression of osteopontin and osteocalcin, the subpopulation found more suitable for the osteogenic differentiation is the one isolated with the immunomagnetic beads coated with anti STRO-1 antibody. The alizarin red staining of the cells isolated with anti STRO-1 ab showed an intense mineralization. In fact, considering the gene expression it is possible to appreciate correspondent low levels of osteopontin and five times higher levels of osteocalcin expression, demonstrating that these cells are in an advanced stage of the osteogenic differentiation [53, 54, 57]. Additionally, the levels of osteocalcin expression of the cells isolated with STRO-1 are lower when compared with the cells isolated with the other antibodies because the differentiation process of the STRO-1+ population have already reached the stage where the extracellular matrix is over produced and the down-regulation of osteocalcin is just beginning [57] (Figs. 3 and 5).

Regarding the chondrogenic differentiation, all the subpopulation isolated showed a chondrogenic potential, as all were found to be positively stained for safranin-O and toluidin and/or expressed sox-9 and collagen II. Similarly to previous statements on the osteogenic differentiation, the results obtained allowed to conclude that not all the cell subpopulations are in the same stage of the chondrogenic differentiation process after 3 weeks of culture. In fact, while all the cells isolated express sox-9, indicating that they are between the stages of prechondrocyte and columnar chondrocyte [56], the ASCs isolated with the immunomagnetic beads coated with anti CD44 and anti STRO-1 antibodies, do not express collagen II showing that these subpopulations are still in an early chondrogenic stage. The ASCs subpopulations that show the highest chondrogenic differentiation potential are those ones isolated with the immunomagnetic beads coated with anti CD105 and the anti CD29 antibody. These subpopulations exhibit a lower expression of the sox-9 gene and higher expression of collagen II, indicating that sox-9 has been down regulated while collagen II is being up-regulated suggesting that, the cells are reaching the stage of prehypertrophic chondrocytes, that is a late chondrogenic differentiation status [55, 56] (Figs. 3 and 5).

The results obtained from this study allow for the conclusion that among all the ASCs subpopulations isolated, the cells isolated with immunomagnetic beads coated with anti STRO-1 ab show the highest osteogenic potential while the ones isolated using immunomagnetic beads coated with anti CD29 ab and anti CD105 ab have the highest chondrogenic differentiation potential.

Furthermore, the results obtained from the induction of the osteogenic and chondrogenic differentiation underline that not all the subpopulations are suitable for both differentiation lineages considered. For example, the cells isolated with anti CD90 ab, anti CD49d ab and anti p75 ab exhibited a high osteogenic differentiation potential, but demonstrated the lowest chondrogenic differentiation potential. On the other hand, the cells isolated with anti CD73 $a b$ exhibited a high chondrogenic differentiation potential and simultaneously the lowest osteogenic potential.

The results obtained in the present study clearly demonstrate that the vascular fraction of the adipose tissue is composed by several stem cell subpopulations that exhibit a common expression profile for some markers, but show clear differences when other markers are considered. Moreover, the data described demonstrated that the cells subpopulations have different in vitro chondrogenic or osteogenic differentiation profiles. In fact, the combination of the results analysed allowed us to select the subpopulation that can have higher potential application in bone and/or cartilage regenerative medicine approaches. In summary and most importantly, this study highlights the importance of studying ASCs subpopulations and opens the way to other research studies that can provide significative further knowledge on ASCs.

Acknowledgments EU funded Marie Curie Actions Alea Jacta Est for a $\mathrm{PhD}$ fellowship. This work was carried out under the scope of the European NoE EXPERTISSUES (NMP3-CT-2004-500283).

\section{References}

1. Zuk, P. A., Zhu, M., Mizuno, H., et al. (2001). Multilineage cells from human adipose tissue: implications for cell-based therapies. Tissue Engineering, 7, 211-228.

2. Gimble, J. M., \& Nuttall, M. E. (2004). Bone and fat: old questions, new insights. Endocrine, 23, 183-188.

3. Li, H. X., Luo, X., Liu, R. X., Yang, Y. J., \& Yang, G. S. (2008). Roles of Wnt/beta-catenin signaling in adipogenic differentiation potential of adipose-derived mesenchymal stem cells. Molecular and Cellular Endocrinology, 291, 116-124.

4. Seda Tigli, R., Ghosh, S., Laha, M. M., et al. (2009). Comparative chondrogenesis of human cell sources in 3D scaffolds. Journal of Tissue Engineering and Regenerative Medicine, 3, 348-360.

5. Sefcik, L. S., Neal, R. A., Kaszuba, S. N., et al. (2008). Collagen nanofibres are a biomimetic substrate for the serum-free osteogenic differentiation of human adipose stem cells. Journal of Tissue Engineering and Regenerative Medicine, 2, 210-220.

6. Lin, J., Lindsey, M. L., Zhu, B., Agrawal, C. M., \& Bailey, S. R. (2007). Effects of surface-modified scaffolds on the growth and differentiation of mouse adipose-derived stromal cells. Journal of Tissue Engineering and Regenerative Medicine, 1, 211-217.

7. Fraser, J. K., Wulur, I., Alfonso, Z., \& Hedrick, M. H. (2006). Fat tissue: an underappreciated source of stem cells for biotechnology. Trends Biotechnol, 24, 150-154. 
8. Castro-Malaspina, H., Ebell, W., \& Wang, S. (1984). Human bone marrow fibroblast colony-forming units (CFU-F). Progress in Clinical and Biological Research, 154, 209-236.

9. Katz, A. J., Tholpady, A., Tholpady, S. S., Shang, H., \& Ogle, R. C. (2005). Cell surface and transcriptional characterization of human adipose-derived adherent stromal (hADAS) cells. Stem Cells, 23, 412-423.

10. Awad, H. A., Wickham, M. Q., Leddy, H. A., Gimble, J. M., \& Guilak, F. (2004). Chondrogenic differentiation of adiposederived adult stem cells in agarose, alginate, and gelatin scaffolds. Biomaterials, 25, 3211-3222.

11. Tholpady, S. S., Llull, R., Ogle, R. C., Rubin, J. P., Futrell, J. W., \& Katz, A. J. (2006). Adipose tissue: stem cells and beyond. Clinics in Plastic Surgery, 33, 55-62. vi.

12. Safford, K. M., Hicok, K. C., Safford, S. D., et al. (2002). Neurogenic differentiation of murine and human adipose-derived stromal cells. Biochemical and Biophysical Research Communications, 294, 371-379.

13. Fujimura, J., Ogawa, R., Mizuno, H., Fukunaga, Y., \& Suzuki, H. (2005). Neural differentiation of adipose-derived stem cells isolated from GFP transgenic mice. Biochemical and Biophysical Research Communications, 333, 116-121.

14. Lee, J. H., \& Kemp, D. M. (2006). Human adipose-derived stem cells display myogenic potential and perturbed function in hypoxic conditions. Biochemical and Biophysical Research Communications, 341, 882-888.

15. Fraser, J. K., Schreiber, R., Strem, B., et al. (2006). Plasticity of human adipose stem cells toward endothelial cells and cardiomyocytes. Nature Clinical Practice Cardiovascular Medicine, 3 (Suppl 1), S33-S37.

16. Strem, B. M., Zhu, M., Alfonso, Z., et al. (2005). Expression of cardiomyocytic markers on adipose tissue-derived cells in a murine model of acute myocardial injury. Cytotherapy, 7, 282-291.

17. Planat-Benard, V., Menard, C., Andre, M., et al. (2004). Spontaneous cardiomyocyte differentiation from adipose tissue stroma cells. Circulation Research, 94, 223-229.

18. Yamamoto, Y., Banas, A., Murata, S., et al. (2008). A comparative analysis of the transcriptome and signal pathways in hepatic differentiation of human adipose mesenchymal stem cells. Febs Journal, 275, 1260-1273.

19. Stock, P., Staege, M. S., Muller, L. P., et al. (2008). Hepatocytes derived from adult stem cells. Transplantation Proceedings, 40, 620-623.

20. Qu, C. Q., Zhang, G. H., Zhang, L. J., \& Yang, G. S. (2007). Osteogenic and adipogenic potential of porcine adipose mesenchymal stem cells. In Vitro Cellular and Developmental BiologyAnimal, 43, 95-100.

21. Ogawa, R., Mizuno, H., Watanabe, A., Migita, M., Hyakusoku, H., \& Shimada, T. (2004). Adipogenic differentiation by adiposederived stem cells harvested from GFP transgenic mice-including relationship of sex differences. Biochemical and Biophysical Research Communications, 319, 511-517.

22. Xu, Y., Malladi, P., Wagner, D. R., \& Longaker, M. T. (2005). Adipose-derived mesenchymal cells as a potential cell source for skeletal regeneration. Current Opinion in Molecular Therapeutics, 7, 300-305.

23. Ogawa, R., Mizuno, H., Watanabe, A., Migita, M., Shimada, T., \& Hyakusoku, H. (2004). Osteogenic and chondrogenic differentiation by adipose-derived stem cells harvested from GFP transgenic mice. Biochemical and Biophysical Research Communications, 313, 871-877.

24. Zuk, P. A., Zhu, M., Ashjian, P., et al. (2002). Human adipose tissue is a source of multipotent stem cells. Molecular Biology of the Cell, 13, 4279-4295.

25. Huang, J. I., Kazmi, N., Durbhakula, M. M., Hering, T. M., Yoo, J. U., \& Johnstone, B. (2005). Chondrogenic potential of progenitor cells derived from human bone marrow and adipose tissue: a patient-matched comparison. Journal of Orthopaedic Research, 23, 1383-1389.

26. Mochizuki, T., Muneta, T., Sakaguchi, Y., et al. (2006). Higher chondrogenic potential of fibrous synovium-and adipose synovium-derived cells compared with subcutaneous fat-derived cells: distinguishing properties of mesenchymal stem cells in humans. Arthritis and Rheumatism, 54, 843-853.

27. Rodbell, M. (1966). The metabolism of isolated fat cells. IV. Regulation of release of protein by lipolytic hormones and insulin. Journal of Biological Chemistry, 241, 3909-3917.

28. Rodbell, M. (1966). of isolated fat cells. II. The similar effects of phospholipase $\mathrm{C}$ (Clostridium perfringens alpha toxin) and of insulin on glucose and amino acid metabolism. Journal of Biological Chemistry, 241, 130-139.

29. Rodbell, M. (1964). Metabolism of Isolated Fat Cells. I. Effects of Hormones on Glucose Metabolism and Lipolysis. Journal of Biological Chemistry, 239, 375-380.

30. Van, R. L., \& Roncari, D. A. (1977). Isolation of fat cell precursors from adult rat adipose tissue. Cell and Tissue Research, 181, 197-203.

31. Van, R. L., Bayliss, C. E., \& Roncari, D. A. (1976). Cytological and enzymological characterization of adult human adipocyte precursors in culture. Journal of Clinical Investigation, 58, 699704.

32. Marin, P., Andersson, B., Ottosson, M., et al. (1992). The morphology and metabolism of intraabdominal adipose tissue in men. Metabolism, 41, 1242-1248.

33. Cowan, C. M., Shi, Y. Y., Aalami, O. O., et al. (2004). Adiposederived adult stromal cells heal critical-size mouse calvarial defects. Nature Biotechnology, 22, 560-567.

34. Gronthos, S., Franklin, D. M., Leddy, H. A., Robey, P. G., Storms, R. W., \& Gimble, J. M. (2001). Surface protein characterization of human adipose tissue-derived stromal cells. Journal of Cellular Physiology, 189, 54-63.

35. Mitchell, J. B., McIntosh, K., Zvonic, S., et al. (2006). Immunophenotype of human adipose-derived cells: temporal changes in stromal-associated and stem cell-associated markers. Stem Cells, 24, 376-385.

36. Solchaga, L. A., Penick, K., Porter, J. D., Goldberg, V. M., Caplan, A. I., \& Welter, J. F. (2005). FGF-2 enhances the mitotic and chondrogenic potentials of human adult bone marrow-derived mesenchymal stem cells. Journal of Cellular Physiology, 203, 398-409.

37. Post, S., Abdallah, B. M., Bentzon, J. F., \& Kassem, M. (2008). Demonstration of the presence of independent pre-osteoblastic and pre-adipocytic cell populations in bone marrow-derived mesenchymal stem cells. Bone, 43, 32-39.

38. Mareddy, S., Broadbent, J., Crawford, R., \& Xiao, Y. (2009). Proteomic profiling of distinct clonal populations of bone marrow mesenchymal stem cells. Journal of Cellular Biochemistry, 106, 776-786.

39. Tormin, A., Brune, J. C., Olsson, E., et al. (2009). Characterization of bone marrow-derived mesenchymal stromal cells (MSC) based on gene expression profiling of functionally defined MSC subsets. Cytotherapy, 11, 114-128.

40. Gronthos, S., \& Zannettino, A. C. (2008). A method to isolate and purify human bone marrow stromal stem cells. Methods in Molecular Biology, 449, 45-57.

41. Gronthos, S., Zannettino, A. C., Hay, S. J., et al. (2003). Molecular and cellular characterisation of highly purified stromal stem cells derived from human bone marrow. Journal of Cell Science, 116, 1827-1835.

42. Satomura, K., Krebsbach, P., \& Bianco, P. (2000). Gehron Robey P. Osteogenic imprinting upstream of marrow stromal cell differentiation. J Cell Biochem, 78, 391-403. 
43. Ho, A. D., Wagner, W., \& Franke, W. (2008). Heterogeneity of mesenchymal stromal cell preparations. Cytotherapy, 10, 320-330.

44. Peiffer, I., Eid, P., Barbet, R., et al. (2007). A sub-population of high proliferative potential-quiescent human mesenchymal stem cells is under the reversible control of interferon alpha/beta. Leukemia, 21, 714-724.

45. Letchford, J., Cardwell, A. M., Stewart, K., et al. (2006). Isolation of C15: a novel antibody generated by phage display against mesenchymal stem cell-enriched fractions of adult human marrow. Journal of Immunological Methods, 308, 124-137.

46. Vogel, W., Grunebach, F., Messam, C. A., Kanz, L., Brugger, W., \& Buhring, H. J. (2003). Heterogeneity among human bone marrow-derived mesenchymal stem cells and neural progenitor cells. Haematologica, 88, 126-133.

47. Sengenes, C., Miranville, A., Maumus, M., de Barros, S., Busse, R., \& Bouloumie, A. (2007). Chemotaxis and differentiation of human adipose tissue CD34+/CD31-progenitor cells: role of stromal derived factor- 1 released by adipose tissue capillary endothelial cells. Stem Cells, 25, 2269-2276.

48. Sengenes, C., Lolmede, K., Zakaroff-Girard, A., Busse, R., \& Bouloumie, A. (2005). Preadipocytes in the human subcutaneous adipose tissue display distinct features from the adult mesenchymal and hematopoietic stem cells. Journal of Cellular Physiology, 205, 114-122.

49. Miranville, A., Heeschen, C., Sengenes, C., Curat, C. A., Busse, R., \& Bouloumie, A. (2004). Improvement of postnatal neovascularization by human adipose tissue-derived stem cells. Circulation, 110, 349-355.
50. Zannettino, A. C., Paton, S., Kortesidis, A., Khor, F., Itescu, S., \& Gronthos, S. (2007). Human mulipotential mesenchymal/stromal stem cells are derived from a discrete subpopulation of STRO1bright/CD34 /CD45(-)/glycophorin-A-bone marrow cells. Haematologica, 92, 1707-1708.

51. Rolf, H. J., Kierdorf, U., Kierdorf, H., et al. (2008). Localization and characterization of STRO-1 cells in the deer pedicle and regenerating antler. PLOS ONE, 3, e2064.

52. Dominici, M., Le Blanc, K., Mueller, I., et al. (2006). Minimal criteria for defining multipotent mesenchymal stromal cells. The International Society for Cellular Therapy position statement. Cytotherapy, 8, 315-317.

53. Stains, J. P., \& Civitelli, R. (2005). Cell-to-cell interactions in bone. Biochemical and Biophysical Research Communications, $328,721-727$.

54. Stains, J. P., \& Civitelli, R. (2005). Cell-cell interactions in regulating osteogenesis and osteoblast function. Birth Defects Research Part C: Embryo Today, 75, 72-80.

55. Goldring, M. B., Tsuchimochi, K., \& Ijiri, K. (2006). The control of chondrogenesis. Journal of Cellular Biochemistry, 97, 33-44.

56. Lefebvre, V., \& Smits, P. (2005). Transcriptional control of chondrocyte fate and differentiation. Birth Defects Research Part C: Embryo Today, 75, 200-212.

57. Lian, J. B., \& Stein, G. S. (1995). Development of the osteoblast phenotype: molecular mechanisms mediating osteoblast growth and differentiation. Iowa Orthopaedic Journal, 15, $118-140$. 